\title{
Young Urologist Research Workshop (YURW)
}

Department of Urology, Kyoto Prefectural University of Medicine ${ }^{1)}$, Department of Urology, Graduate School of Medicine, Chiba University ${ }^{2)}$

Yoichi Mizutani' ${ }^{1)}$, Tomohiko Ichikawa ${ }^{2)}$

Dear Colleagues :

The exciting progress in urology has been made in all areas of basic, translational and clinical research recently. New technology and the following dramatic advances in biological information have led to a significantly improved diagnosis and treatment for urological diseases. Professor Akihiko Okuyama, the president of the 92nd Annual Meeting of the Japanese Urological Association, and 10 program committees have planned an outstanding program, Young Urologist Research Workshop (YURW). Our exiting challenge has been to evolve the program. YURW promises to be exceptional. In ordinary annual meetings, most of speakers in symposia and workshops are full/associate/assistant professors. The YURW program is specifically designed for young urologists (less than forty years old). Young urologists will participate in YURW as speakers as well as discussants. The language used is English. World-wide eminent scientists are being scheduled to attend YURW as commentators. Of course, YURW will be highlighting new data in each of emerging and rapidly growing research areas. When we invited young urologists to submit their abstracts for YURW, we received approximately eighty excellent abstracts. Fiftytwo significant abstracts were selected by the program committees.

YURW will assist you in integrating the growing scientific information into your research efforts. In addition, it will provide an ideal forum for the discussion of research results by young urologists as well as experts in various urological fields, with the goal of speedy delivery of useful diagnostic tools and effective treatments to the clinic. Furthermore, YURW will generate new ideas and will help new collaborations for your future research. Moreover, we believe that young urologists will have valuable opportunities and will be encouraged very much in YURW.

YURW will be held in the morning for three days (from April 10 to April 12, 2004). Please attend YURW and share our latest results. We look forward to meeting you in YURW and to discussing the newest research findings and innovative strategies for the future. 